\title{
Análisis numérico comparativo del comportamiento a fatiga de dos osteosíntesis utilizadas en el tratamiento de la fractura de cadera 31 A1 .1.
}

\section{Comparative numerical analysis of the fatigue behavior of two osteosynthesis used in the treatment of hip fracture 31 A1 .1.}

\author{
Ramos-Botello Yoan Manuel \\ Universidad de Granma \\ Facultad de Ciencias Técnicas \\ Departamento de Ingeniería Mecánica \\ Correo: yoanramosbotello@yahoo.com \\ Arias-Hidalgo René Misael \\ Universidad de Granma \\ Facultad de Ciencias Técnicas \\ Departamento de Ingeniería Mecánica \\ Correo: rariash@udg.co.cu
}

\author{
Bosch-Cabrera Jorge Alexander \\ Universidad de Granma \\ Facultad de Ciencias Técnicas \\ Departamento de Ingeniería Mecánica \\ Correo: jbosch@udg.co.cu \\ Estrada-Cingualbres Roberto Andrés \\ Universidad de Holguín "Oscar Lucero Moya" \\ Centro de Estudios CAD/CAM \\ Correo: roberto@cadcam.uho.edu.cu
}

\section{Resumen}

En la presente investigación se simula el comportamiento a fatiga mediante un software de cálculo por elementos finitos, de los fijadores internos placa DHS (Dynamic Hip Screw) y un clavo Gamma, utilizados en el tratamiento de fracturas de cadera. Para el análisis de dicho comportamiento se realizaron estudios estáticos, donde las variables fundamentales a evaluar fueron las tensiones de von Mises. Para ello, se tomó en cuenta la acción de los músculos y el peso corporal para cuatro instantes del ciclo de marcha, así como las restricciones, condiciones de contacto entre los modelos y propiedades mecánicas de cada uno de los mismos. Tomando como base los resultados de los estudios estáticos y las curvas S-N del material de cada modelo, se realizaron estudios de fatiga para determinar la vida en ciclos de cada fijador. Los estudios permitieron determinar dónde son mayores las tensiones y cuál de ellos podrá soportar mayores ciclos de carga antes del fallo por fatiga. En función de dichos resultados, se determinó que el clavo Gamma es el de mejor comportamiento mecánico para todos los casos estudiados, lo que se comprobó con los resultados obtenidos por otros autores. Ya que en los estudios no se consideró la zona trabecular del fémur, los resultados obtenidos solo tienen validez desde el punto de vista cualitativo.

Descriptores: elementos finitos, biomecánica, fracturas de caderas, placa DHS, clavo Gamma.

\begin{abstract}
In the present research the fatigue behavior is simulated with a thin-element calculus software, internal fixing plate DHS (Dynamic Hip Screw) and a Gamma nail, used in the treatment of hip fracture. For the analysis of the behavior static studies have been carried out, where the fundamental variables have been evaluated in the stresses of von Mises. For this, the action of muscles and body weight for four instants of the gait cycle has been taken into account, as well as the restrictions, the contact conditions between the models and the mechanical properties of each one of them. Based on the results of the static studies and the S-N curves of the material of each model, fatigue studies were performed to determine the cycle life of each fixative. The studies allowed to determine where the tensions are greater and which of them can withstand greater cycles of load before the fall by fatigue. Based on the results of the results, it was possible to determine that the Gama nail is the best mechanical behavior, for all the cases studied, which could be verified with the results obtained by other authors. However, since the studies do not consider the trabecular area of the femur, the results obtained are valid only from the qualitative point of view.
\end{abstract}

Keywords: finite elements, biomechanics, hip fracture, plate DHS, Gamma nail. 


\section{INTRODUCCIÓN}

Debido al creciente número de fracturas pertrocantéreas, resulta de gran importancia el estudio detallado de todo lo concerniente al tratamiento de las mismas. Son las osteosíntesis extramedulares e intramedulares los métodos actuales para la fijación de este tipo de fractura. En la actualidad no existe un dispositivo ideal a emplear, aunque son muy utilizados el tornillo-placa deslizante y el clavo Gamma. Existen varias causas que provocan el fracaso del tratamiento de este tipo de fractura, entre las que se encuentran el llamado stress-shielding, que no es más que la disminución de las tensiones que soporta el hueso subyacente al implante, ya que este último es más rígido y las absorbe. El material óseo responde a la tensión remodelándose según el nivel de esfuerzo aplicado. Debido al stress-shielding, el hueso se remodela a un nivel de carga más bajo que el que debería soportar, produciéndose un proceso de osteopenia o pérdida de la masa ósea alrededor del implante, lo que provoca perdida de estabilidad en dicho implante, apareciendo importantes micromovimientos bajo cargas funcionales (Tavakkoli, 2015).

Otra de las causas del fracaso del tratamiento de estas fracturas es la que se conoce como falla mecánica, que muchas veces se manifiesta por la rotura del implante. Este tipo de rotura se debe fundamentalmente a la acción de carga y descarga de los miembros inferiores durante las actividades cotidianas desarrolladas por las personas, influyendo además, el medio altamente corrosivo al que se ven sometidas las osteosíntesis en el cuerpo humano (Ramos, 2013; Vigil, 2011). Este tipo de falla se conoce como falla por fatiga.

En investigaciones como la de Estrada (2014) se realizan estudios comparativos entre dos fijadores de cadera. Si bien en dicha investigación se analizan las tensiones en los fijadores, esta se centra en la distribución del estado tensional en el fémur, antes, durante y después de retirado el implante. Otras investigaciones como las de Chang (2015); Chen (2014); Matityahu (2013) también realizan estudios mediante elementos finitos, analizando el estado tensional de fijadores internos. De forma general, todas estas investigaciones han obtenido resultados acertados para el estado de carga considerado. La limitación de dichas investigaciones se encuentra en que solo se utiliza un estado de carga, lo que limita el alcance de los resultados. En un gran número de investigaciones solo se estudian las tensiones en los fijadores, tal como es el caso de las referenciadas anteriormente. Sin embargo, en la mayoría de los casos, las fallas mecánicas en los fijadores de cadera se deben a la rotura por fatiga. Según las investigaciones publicadas, los cálculos numéricos a fijadores de cadera, se realizan a dispositivos que ya se han probado en la práctica, por lo que al realizar los cálculos de las tensiones, estas darán valores aceptables y se concluye que el diseño es seguro. Este tipo de estudio obvia que cada ciclo de fluctuación de tensión debilita al fijador hasta cierto punto y después de una cantidad de ciclos es muy probable que dicho fijador falle. Por tal motivo, en esta investigación se pretenden realizar estudios de fatiga, a partir de estudios estáticos calculados, teniendo en cuenta cuatro estados de carga diferentes, correspondientes a cuatro instantes del ciclo de marcha.

La presente investigación tiene como objetivo, además, analizar mediante elementos finitos, el comportamiento a fatiga de los sistemas de fijación interna, placa DHS y el clavo Gamma, utilizados en el tratamiento de la fractura de la región trocantérea 31A1.1 según la clasificación de la Arbeitsgemeinshaft für Osteosynsthesefragen $(\mathrm{AO})$.

\section{DesarRollo}

Los modelos en 3D de los fijadores se obtuvieron con la ayuda de un software de Diseño Asistido por Computadora (CAD), a partir de la minuciosa medición de cada detalle de fijadores reales. El modelo del fémur se obtuvo a través de una Tomografía Axial Computarizada (TAC). Dicho fémur es izquierdo con una longitud máxima de $450 \mathrm{~mm}$ y el diámetro de la cabeza de $51.20 \mathrm{~mm}$. En el mismo no se contempló el hueso trabecular, lo que representa peores condiciones para el modelo respecto a las reales.

El ángulo de $130^{\circ}$ de los dispositivos corresponde con el ángulo entre los ejes de la diáfisis y el cuello femoral del modelo del fémur. La fractura 31A1.1 se simuló habiendo estudiado previamente varias radiografías de este tipo de fracturas, lo que permitió definir con bastante precisión la zona y forma de la misma. El ensamble de cada elemento se realizó cuidando que no hubiese interferencias entre componentes que pudiesen introducir errores en los cálculos (figura 1).

\section{CARGAS Y RESTRICCIONES}

Desde el punto de vista biomecánico, los músculos del fémur transmiten fuerzas en diferentes direcciones según la actividad física que se realice, por lo que no se puede tratar la biomecánica del fémur sin tomar en cuenta la acción de dichos músculos, que son los que permiten un determinado movimiento como caminar, subir escaleras o correr (Estrada, 2014.; Ojeda, 2009). Sobre la magnitud de las fuerzas musculares ejercidas so- 
bre el fémur, no existe pleno consenso, pero sin lugar a dudas, son las investigaciones de Bergmann (2001) y Heller (2001) las más referenciadas en la literatura especializada y con los que se han obtenidos resultados más acertados.

De las actividades físicas que realiza una persona en su vida cotidiana, caminar es la más común, por lo que en esta investigación se consideran las fuerzas musculares que se ejercen durante la misma. En muchas investigaciones a la hora de elegir el estado de carga, se limitan solo a un instante del ciclo de marcha, generalmente la fase monopodal. Las investigaciones realizadas teniendo en cuenta esta simplificación, obvian por completo los efectos que puedan ocasionar sobre el implante el resto de los estados de carga a lo largo de dicho ciclo. Estas simplificaciones se deben fundamentalmente a lo extremadamente complicado de tener en cuenta en un análisis, todas las cargas que se producen por el efecto corporal sobre el fémur durante el ciclo de marcha.

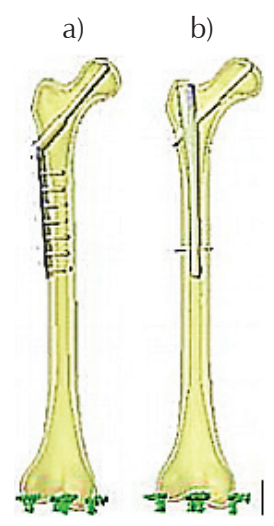

Figura 1. Sistemas de fijación interna, a) placa DHS, b) clavo Gamma
Estos aspectos son particularmente importantes a la hora de realizar estudios de fatiga, ya que el efecto de las cargas en los dispositivos de fijación en cada instante del ciclo de marcha es acumulativo e irreversible.

Para el estudio a realizar se consideró el efecto de una persona de $85 \mathrm{~kg}$ y del ciclo de marcha, se escogieron cuatro instantes correspondientes a $25 \%, 45 \%, 65 \%$ y $85 \%$ de dicho ciclo, coincidiendo estos con los estudios realizados por Behrens (2009). Todas las fuerzas musculares y las correspondientes a las del peso corporal para cada uno de los cuatro instantes del ciclo, se descompusieron en las componentes de los tres ejes de coordenadas. Los valores de estas fuerzas se muestran en la tabla 1 .

La zona de inserción de los músculos en el fémur, donde se aplicarán las componentes de las fuerzas correspondientes a cada uno de los casos a analizar, así como el punto donde aplicará la fuerza correspondiente al peso corporal, se muestra en la figura 2. Para la determinación de la zona donde se aplicarán las fuerzas musculares se tomaron en cuenta las investigaciones de Bayoglu (2015) y Netterl (2011).

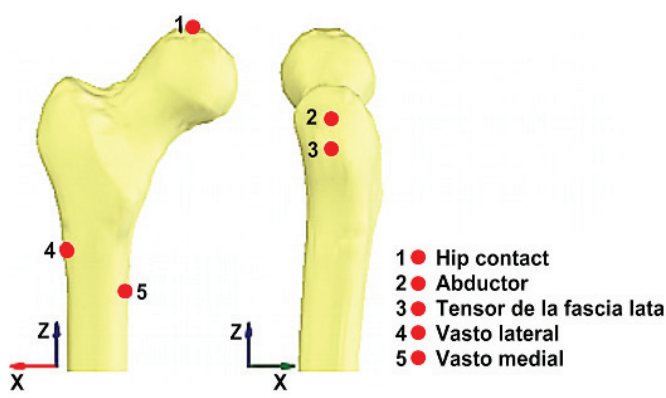

Figura 2. Ubicación de las fuerzas

Tabla 1. Fuerzas musculares y del peso corporal (Behrens, 2009; Bergmann, 2001; Heller, 2001)

\begin{tabular}{|c|c|c|c|c|c|c|c|c|c|c|c|c|c|}
\hline \multirow{3}{*}{ Carga } & & \multicolumn{12}{|c|}{ Fuerzas actuantes durante el ciclo de marcha } \\
\hline & & \multicolumn{2}{|l|}{$25 \%$} & \multicolumn{3}{|c|}{$45 \%$} & \multicolumn{3}{|c|}{$65 \%$} & \multicolumn{3}{|c|}{$85 \%$} & \multirow[b]{2}{*}{$\mathrm{z}$} \\
\hline & & $\mathrm{x}$ & $\mathrm{y}$ & z & $\mathrm{x}$ & $\mathrm{y}$ & z & $\mathrm{x}$ & $\mathrm{y}$ & z & $\mathrm{x}$ & $\mathrm{y}$ & \\
\hline Hip contact & $(\mathrm{N})$ & 451.4 & 225.7 & -1806 & 393 & -41.8 & -1663 & 334.4 & -58.5 & -786 & -108 & -16.7 & -251 \\
\hline Abductor & $(\mathrm{N})$ & -468 & 0 & 694 & -334 & -92 & 501.6 & 0 & 0 & 0 & 0 & 0 & 0 \\
\hline Tensor fascia latae & $(\mathrm{N})$ & 117 & 158.8 & -75.2 & 142 & 158.4 & -58.5 & 0 & 0 & 0 & 8.4 & 16.7 & 0 \\
\hline Vastus lateralis & $(\mathrm{N})$ & 8.4 & -108 & -543 & 0 & 0 & 0 & 0 & 0 & -25 & 0 & 0 & 0 \\
\hline Vastus medialis & $(\mathrm{N})$ & 8.4 & -33.4 & -167 & 0 & 0 & 0 & 0 & 0 & -8 & 0 & 0 & 0 \\
\hline
\end{tabular}


Por la complejidad de representar en los estudios la restricción al movimiento del fémur debido al efecto del conjunto de músculos y la articulación de la rodilla, para cada uno de los casos de estudio se trabajó con un modelo más simple, donde se consideró al fémur empotrado en la epífisis inferior. Esta simplificación se ha utilizado en otras investigaciones, obteniéndose resultados acertados, tales como los de Bayoglu (2015) y Ramos (2013).

\section{Propiedades mecánicas de los materiales}

Determinar las propiedades mecánicas del material óseo, ha sido objeto de estudio de muchos investigadores, quienes han utilizado distintas técnicas experimentales, basadas en métodos mecánicos o utilizando las técnicas de ultrasonidos. De acuerdo con estas investigaciones se ha demostrado que el comportamiento del tejido óseo es anisótropo, viscoelástico y no lineal (Geraldes, 2014; Buroni, 2004). Debido a las diferentes técnicas utilizadas para determinar dichas propiedades y a que el material óseo se encuentra en permanente cambio según sean los estímulos que reciba, existe diversidad en cuanto a los valores de las propiedades obtenidas de acuerdo con los diferentes investigadores. Por este motivo, muchos autores para simplificar los estudios consideran al hueso como un material elástico-lineal, lo que se aproxima razonablemente a la realidad, salvo en el caso de cargas de impacto. Sin embargo, el hecho de considerar un material con estas características, cuando realmente se trata de un material anisótropo, puede dar lugar a errores que en algunos casos pueden ser significativos. En esta investigación se considera el material de la zona cortical del fémur como anisótropo, después de haber revisado, analizado y calculado la media de las propiedades mecánicas a partir de los datos expuestos al respecto por Pise (2012); Rellán (2012); Trabelsi (2011); Müller (2001), quienes definieron el material con esta característica. En la tabla 2 se muestran las propiedades mecánicas obtenidas.

Los biometales tienen características específicas que los hacen apropiados para su utilización en el cuerpo humano. Lo primero que deben garantizar estos materiales es su estabilidad química, resistencia a la corrosión y no ser carcinogénico ni tóxico cuando se usa en el cuerpo humano. Lo segundo que deben garantizar es la capacidad de soportar tensiones elevadas y variables durante su vida útil (Smith, 2006). Entre los biometales más utilizados para la fabricación de los sistemas de fijación interna objeto de estudio, se encuentra el acero quirúrgico AISI 316L y el titanio Ti-6AI-4V. Estas propiedades mecánicas de materiales se utilizan en esta investigación, fueron tomadas de la investigación realizada por Samiezadeh (2014) y Mohammad (2013). En la tabla 3 se muestran dichas propiedades.

\section{Curvas S-N}

La fatiga es la causa fundamental de rotura de muchas piezas que se someten a cargas cíclicas. En el caso particular de los sistemas de fijación interna, el proceso cíclico de carga y descarga provocado por la acción muscular y el peso corporal durante la marcha puede originar este tipo de falla (Matityahu, 2013). El material óseo tiene la capacidad de soportar cargas que varían de acuerdo con las actividades físicas desarrolladas y resistirse a la fractura. Sin embargo, pueden surgir microfisuras como consecuencia de la fatiga en el hueso. Aunque a diferencia de otros materiales, el material óseo tiene la capacidad de reparar esas microlesiones (Trębacz, 2013; Taylor, 1998). En muchas investigaciones se exponen las propiedades mecánicas de dicho material, obtenidas desde el punto de vista de la estática, no así con las concernientes a las de fatiga. Esto se debe en gran medida a lo complicado de obtener probetas de este material con las características necesarias
Tabla 2. Propiedades mecánicas del material del fémur

\begin{tabular}{llcc}
\hline \multicolumn{2}{c}{ Nomenclatura } & Nombre & Valor \\
\hline $\mathrm{E}_{X}$ & $(\mathrm{GPa})$ & Módulo elástico X & 11.543 \\
$\mathrm{E}_{Y}$ & $(\mathrm{GPa})$ & Módulo elástico $\mathrm{Y}$ & 14.845 \\
$\mathrm{E}_{Z}$ & $(\mathrm{GPa})$ & Módulo elástico Z & 22.365 \\
$\mathrm{G}_{X Z}$ & $(\mathrm{GPa})$ & Módulo cortante & 6.0225 \\
$\mathrm{G}_{X Y}$ & $(\mathrm{GPa})$ & Módulo cortante & 5.0550 \\
$\mathrm{G}_{Z Y}$ & $(\mathrm{GPa})$ & Módulo cortante & 7.0025 \\
$\mu_{X Y}$ & Coeficiente de Poisson & 0.4087 \\
$\mu_{X Z}$ & Coeficiente de Poisson & 0.2540 \\
$\mu_{Z Y}$ & Coeficiente de Poisson & 0.2295 \\
\hline
\end{tabular}

Tabla 3. Propiedades mecánicas del acero quirúrgico AISI 316L y el titanio Ti-6Al-4V

\begin{tabular}{lccc}
\hline Nomenclatura & Nombre & AISI 316L & Ti-6AI-4V \\
\hline E $(\mathrm{GPa})$ & Módulo elástico & 165 & 113.8 \\
$\sigma \mathrm{e}(\mathrm{MPa})$ & Límite elástico & 332 & 790 \\
$\sigma \mathrm{T}(\mathrm{MPa})$ & Límite de rotura a tracción & 673 & 860 \\
$\mu \quad$ Coeficiente de poisson & 0.28 & 0.34 \\
$\mathrm{Q} \quad(\mathrm{kg} / \mathrm{m} 3)$ & Densidad & 8000 & 4430 \\
\hline
\end{tabular}


para este tipo de pruebas. Para este estudio se tomó la curva de tensión contra número de ciclos (S-N) obtenida por Swanson (1971) para el material de la región cortical del fémur, que se muestra en la figura 3.

Cualquier dispositivo de fijación interna utilizado en el tratamiento de las fracturas proximales del fémur, corre el riesgo de sufrir rotura por fatiga, debido a las repetidas combinaciones de solicitaciones que surgen en los miembros inferiores. Para los estudios a realizar, se consideró la curva S-N del acero AISI 316L y el titanio Ti-6AI-4V, según los estudios de Mohammad (2013) y Kayabasi (2008) respectivamente, las cuales se muestran en la figura 4.

Para realizar los cálculos a fatiga, es necesario obtener los valores de tensiones que se producen en los modelos producto de los cuatro estados de carga. Para este estudio se utilizaron como tensiones principales, las tensiones de von Mises, a partir de las cuales se calcularon las tensiones alternas, las tensiones medias y la relación de tensiones (ecuaciones 1, 2 y 3, respectivamente). Estas variables conjuntamente con las curvas $\mathrm{S}-\mathrm{N}$ de los materiales se utilizaron para obtener los números de ciclos a los que puedan causar fallo los modelos.

$$
\sigma_{a}=\frac{\sigma_{\text {máx }}-\sigma_{\min }}{2}
$$

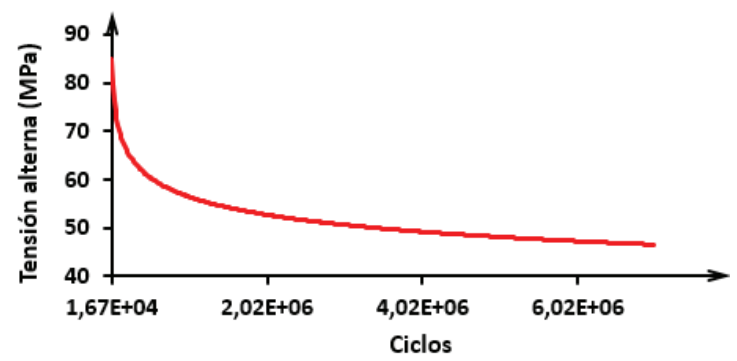

Figura 3. Curva S-N del material del fémur

a)

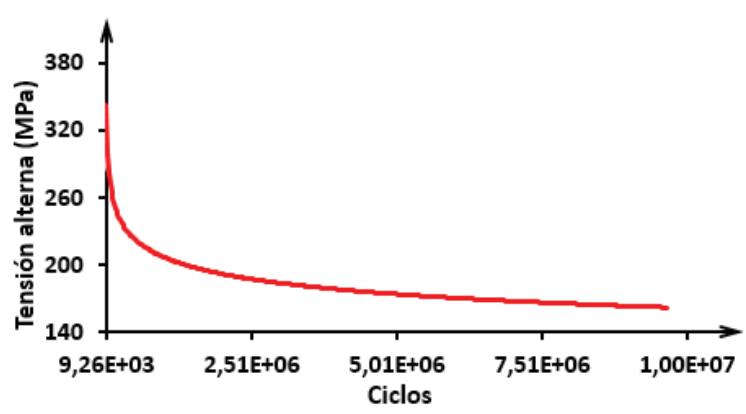

Figura 4. Curvas S-N, a) Acero AISI 316L, b) Titanio Ti-6AI-4V $\sigma_{m}=\frac{\sigma_{\text {máx }}-\sigma_{\min }}{2}$

$R=\frac{\sigma_{\min }}{\sigma_{\operatorname{máx}}}$

$\sigma_{\text {máx }}$ Tensión máxima

$\sigma_{\text {min }}:$ Tensión mínima

Ya que cada fijador se somete a cuatro estados de cargas correspondientes: $25 \%$, 45\%, 65\% y $85 \%$ del ciclo marcha, cada uno de estos casos será considerado como un suceso de fatiga y la evaluación de las tensiones alternas se harán mediante interacción aleatoria, lo que predice un factor de daño más alto que la aplicación consecutiva de sucesos de fatiga.

Una vez realizado, se empleará la regla de daño lineal o regla de Palmgren-Miner's para determinar el daño causado por dichos ciclos (ecuación 4). Esta regla supone que los ciclos de tensión con una tensión alterna que supere el límite de resistencia, causará un daño permanente que se puede medir. Además, plantea que el daño total causado por una cantidad de ciclos de tensión, es igual a la suma de los daños causados por los ciclos de tensión individuales. Si bien esta regla no toma en cuenta los efectos del orden en el cual se aplican los esfuerzos, se emplea con frecuencia debido a su sencillez y al hecho de que al comparar los resultados obtenidos con esta y con los de otras teorías más complejas, no siempre se obtienen diferencias significativas (Chen, 2011).

$$
D=\sum_{i=1}^{k} \frac{n_{i}}{N_{i}}
$$

donde

$n_{i}=$ Número ciclos a una tensión alterna $\sigma_{i}$

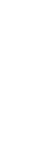


$N_{i}=$ Ciclos necesarios para causar un fallo bajo la tensión $\sigma_{i}$

$D=$ Daño total

\section{Mallado}

Para los cálculos realizados por el método de elementos finitos, es importante escoger adecuadamente el tipo de elemento, así como el tamaño y tolerancia de este, ya que de ello depende la discretización del dominio tridimensional, que debe satisfacer condiciones estrictas para conseguir predicciones confiables (Ojeda, 2009). A todos los modelos analizados en esta investigación se les realizó un mallado global con elementos finitos tipo sólido tetraédrico de alto orden (10 nodos), con tres grados de libertad por nodo. En el caso de los fijadores, se mallaron con un tamaño de elementos de $3 \mathrm{~mm}$ y una tolerancia $0.1 \mathrm{~mm}$. En cambio, el modelo del fémur se malló con un tamaño de elementos de $6 \mathrm{~mm}$ y la misma tolerancia. Además, se aplicó un control de mallado en la zona de unión entre los tornillos con las bases de los fijadores y en la unión de los mismos con el fémur.

El tipo de elemento utilizado, tal como lo plantea Ramos (2006) y Viceconti (1998) se adapta adecuadamente al tipo de estudio y a modelos que presentan grandes irregularidades geométricas como los huesos.

El tipo de contacto utilizado entre los fijadores y el fémur, es de tipo superficie a superficie, sin penetración. Este evita las interferencias entre las caras de origen y destino durante la aplicación de las cargas. Este tipo de contacto permite que las caras en contacto puedan separarse e impide que los modelos se introduzcan unos dentro de otros, posibilitando que los resultados obtenidos sean lo más acertados posible. Para el caso de la fractura se ha supuesto que ha transcurrido el tiempo suficiente para que haya ocurrido la formación de callo óseo. Para simular este proceso se definió un tipo de contacto de unión rígida entre las partes del fémur. Una vez realizado el mallado de los modelos, se obtuvieron 31789 elementos y 53816 nodos para el caso del fémur con la placa DHS, mientras que para el modelo del fémur con el clavo Gamma se obtuvieron 25930 elementos con 43726 nodos.

\section{DisCUSIÓN Y ANÁLISIS DE RESULTADOS}

\section{PLACA DHS}

En la figura 5 se muestra la distribución de tensiones de von Mises que se producen en la placa DHS para los cuatro instantes del ciclo de marcha. En el caso de $25 \%$ los máximos valores de tensiones se producen en la zona de contacto del último tornillo de cortical, con la placa con un valor de $255.8 \mathrm{MPa}$ mientras que para $45 \%$ fueron de 182.5 MPa en la unión del tornillo intramedular con la placa. Esta diferencia en cuanto a las tensiones se debe a que para $45 \%$ del ciclo de marcha los valores de cargas son menores, además, la acción de los músculos vasto lateral y vasto medial es despreciable. En el caso de 65 y $85 \%$ de dicho ciclo, los valores de tensiones fueron de 121.9 MPa y 72.5 $\mathrm{MPa}$, respectivamente, coincidiendo la zona de máximos valores de tensiones con la de $25 \%$. En estos dos últimos casos los valores de las fuerzas ejercidas por los músculos y el efecto del peso corporal van disminuyendo, lo que explica la disminución de los valores de las tensiones. Al comparar el máximo valor de tensión con el límite elástico del material de la osteosíntesis, se observa que dicho valor de tensión es 1.3 veces menor, lo que demuestra que las deformaciones no serán permanentes.

\section{Clavo Gamma}

En el caso del clavo Gamma, los máximos valores de tensiones se producen en la ranura donde se coloca el tornillo de bloqueo distal y en el propio tornillo, para los cuatro instantes del ciclo analizado (figura 6). Al igual que para el caso de la placa DHS, en el clavo Gama, los máximos valores de tensiones se observan para $25 \%$ con en valor máximo de $143.1 \mathrm{MPa}$ mientras que para los otros casos analizados los valores de tensiones fueron de 71.3 $\mathrm{MPa}$, 89.6 $\mathrm{MPa}$ y $51.78 \mathrm{MPa}$, respectivamente. Los máximos valores de tensiones se producen en esta zona debido a que las cargas sobre el fémur, se contrarrestan en gran medida en la zona de unión entre estos dos elementos, ya que el tornillo de bloqueo distal se colocó de forma que permite un bloqueo estático y, a su vez, impide la rotación del sistema. Esta forma de colocación del tornillo de bloqueo provocó fundamentalmente solicitaciones de cizallamiento, lo que puede provocar su rotura. No obstante, al comparar las tensiones obtenidas en el estudio con el límite elástico del titanio Ti6AI4V, se puede ver que dichos valores de tensiones están por debajo del límite elástico, estando el máximo valor 5.5 veces por debajo de dicho límite.

\section{Comparación ENTRE los VALORES DE TENSIONES}

En la figura 7 se muestra un gráfico donde se exponen los máximos valores de tensiones en los fijadores objeto de estudio para los cuatro instantes del ciclo de marcha analizado. Se puede apreciar que en los cuatro casos, los máximos valores se produjeron en la placa DHS, 


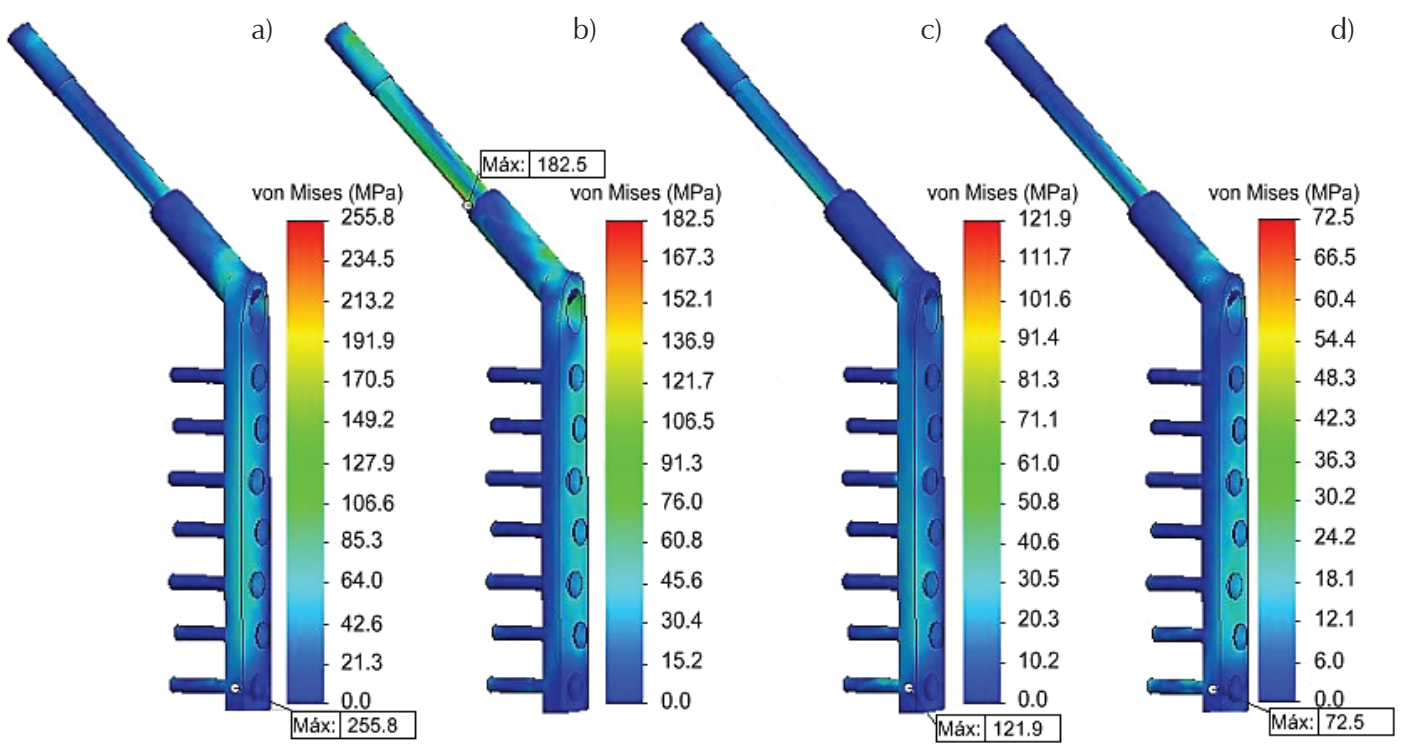

Figura 5. Tensiones de von Mises en la placa DHS, a) 25\% del ciclo, b) 45\% del ciclo, c) 65\% del ciclo, d) $85 \%$ del ciclo

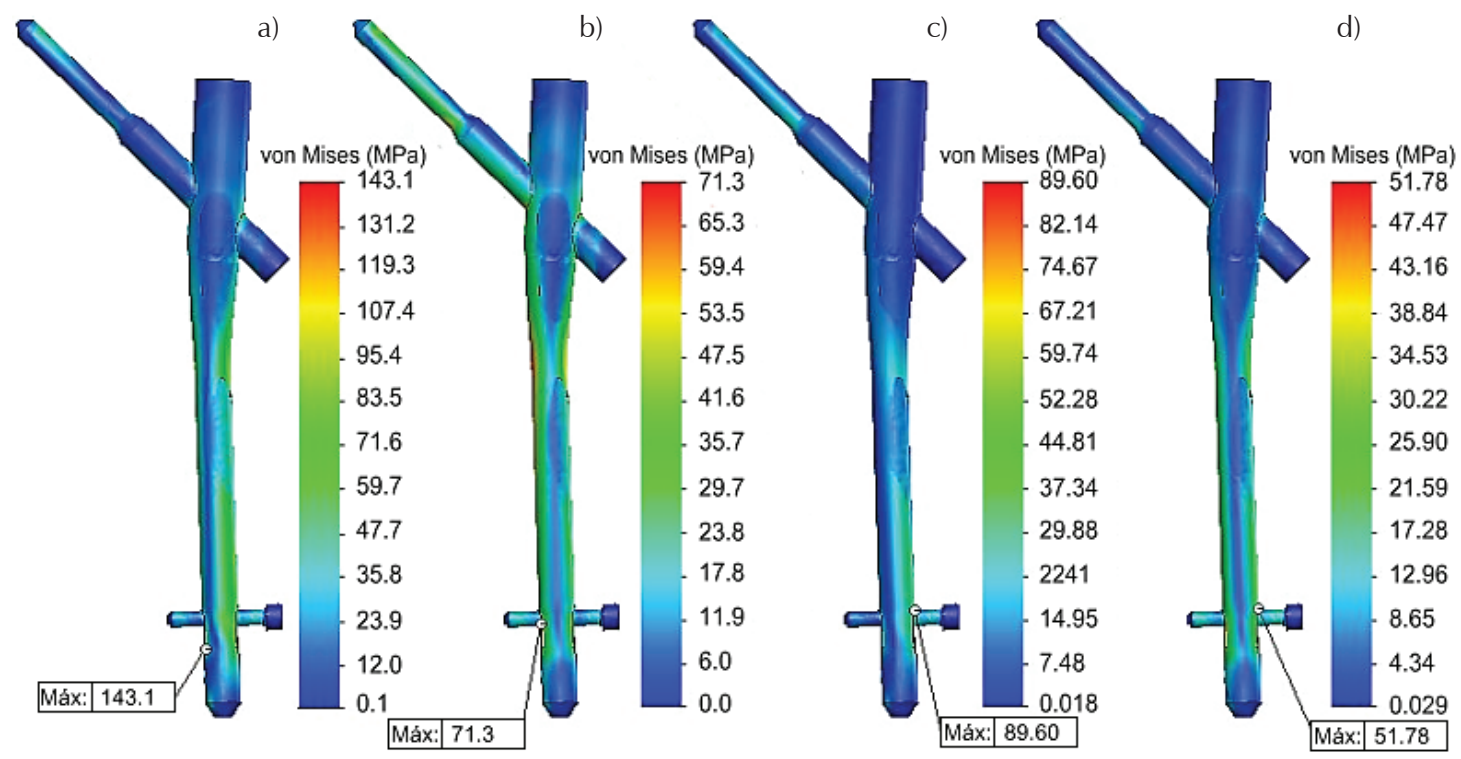

Figura 6. Tensiones de von Mises en el clavo Gamma, a) 25\% del ciclo, b) $45 \%$ del ciclo, c) $65 \%$ del ciclo, d) $85 \%$ del ciclo

que pone en evidencia que el clavo Gamma tiene mejor comportamiento mecánico desde el punto de vista del estado tensional, lo cual coincide con los obtenidos por Seral (2001), quien realiza estudios similares a este. Los máximos valores de tensiones en los casos analizados se producen en los tornillos, que son los que con más frecuencia se rompen en la práctica (Orozco, 2001). Una de las causas que puede provocar elevados valores de tensiones en los componentes de los fijadores, es cuando no existe contacto entre las partes del hueso a unir o es muy poco, lo cual provoca que el fijador absorba todas o casi en su totalidad las solicitaciones.

\section{DEFORMACIONES}

Al analizar las deformaciones en los dos fijadores (figura 8), se observa que los máximos valores se producen en la zona donde fueron máximos los valores de tensiones. Esto se debe a que los estudios realizados fueron lineales y los materiales de los fijadores cumplen con la 
ley de Hooke y los valores de deformación están dentro de la zona elástica. Se puede considerar que los valores de tensiones no superan los límites de proporcionalidad. En la figura 8 se observan las deformaciones para $25 \%$ del ciclo de marcha, que en este caso, es donde son mayores las tensiones y deformaciones en ambos fijadores.

\section{ANÁLISIS DE FATIGA}

Al analizar los resultados de vida en ciclos para las dos osteosíntesis, se puede observar que para el estado tensional al que fueron sometidas en los cuatro instantes del ciclo estudiado, ninguna de ellas causa fallo por fatiga hasta después del millón de ciclos. En el caso de la placa DHS esto sucederá a partir de los 1,832 millones de ciclos. En este fijador el elemento que primero fallará será el último tornillo de cortical seguido del penúltimo

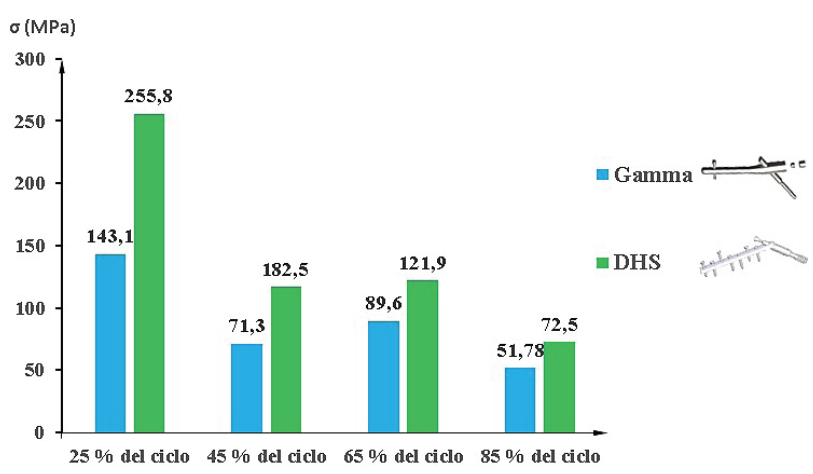

Figura 7. Máximos valores de tensión en los fijadores clavo Gamma y placa DHS para los cuatro instantes del ciclo de marcha

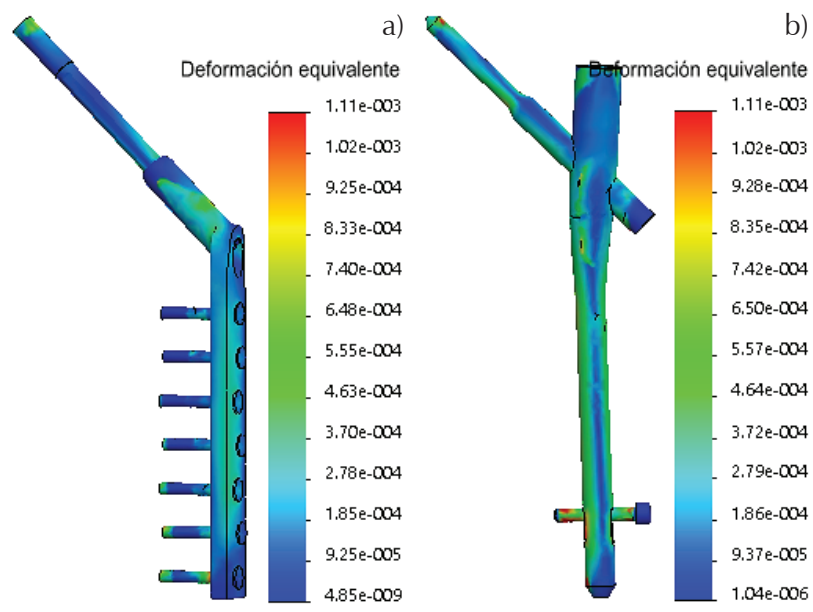

Figura 8. Deformación equivalente para 25\% del ciclo, a) placa DHS, a) clavo Gamma tornillo, elementos donde se observaron los mayores valores de tensiones (figura 9a). En el caso del clavo Gamma el comportamiento a fatiga fue mejor que en el caso de la placa DHS. El menor número de ciclos a partir de los cuales, los elementos causarán fallo es de 3,539 millones. En este fijador el elemento con más peligro de rotura por fatiga es el tornillo de bloqueo, ya que se somete a tensiones de corte. En el clavo endomedular también se observan zonas peligrosas, principalmente alrededor del orificio de entrada del tornillo deslizante, lugar donde la sección del clavo se reduce aproximadamente $70 \%$ (figura $9 b$ ).

\section{VALIDACIÓN DE LOS RESULtAdOS}

Para validar los resultados obtenidos y comprobar que son razonables, se comprobó la existencia de un equilibrio de fuerzas entre cargas aplicadas y reacciones en los apoyos. Al analizar el error existente entre las fuerzas aplicadas y las reacciones correspondientes dio como resultado un valor de 0.01 por lo que se puede decir que dicho equilibrio se cumplió y que los resultados de tensiones obtenidos se encuentran en lo razonable. Para validar la precisión de las tensiones, se analizó la relación existente entre el tamaño de los elementos y el rango de error de los resultados. Para ello se redujo el tamaño global de los elementos a la mitad y se obtuvieron los valores de tensiones para cada caso. El error máximo obtenido fue de 0.4 por lo que se puede decir que los resultados obtenidos son válidos y razonables, ya que no sufrieron cambios significativos al reducir el tamaño de los elementos en el mallado.

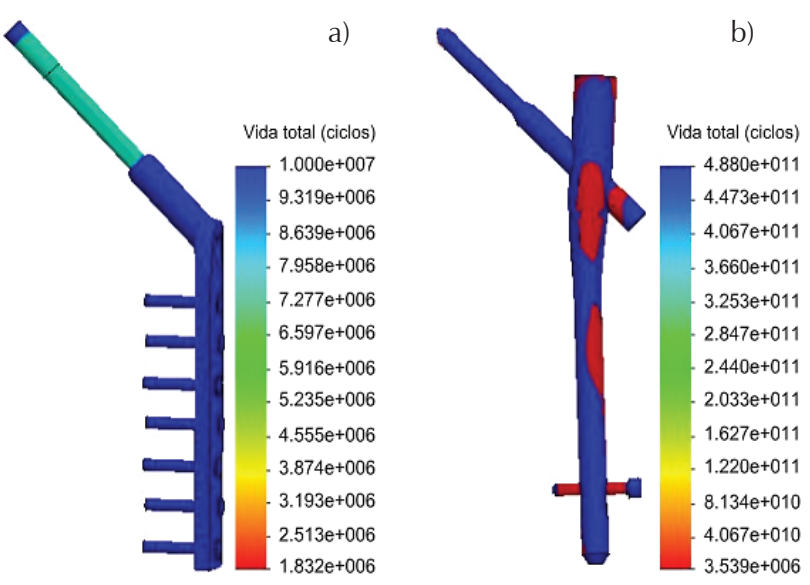

Figura 9. Resultados del estudio a fatiga, a) placa DHS, b) clavo Gamma 


\section{Conclusiones}

A partir de los resultados del estudio de las tensiones se pudo determinar que el comportamiento del clavo Gamma es más favorable que el de la placa DHS, ya que en esta última osteosíntesis se observaron los mayores valores de las mismas. No obstante, para todos los estudios realizados, los valores de tensiones están por debajo del límite de fluencia de los materiales de las osteosíntesis, lo que está en correspondencia con los resultados de los estudios de las deformaciones, que no mostraron valores significativos.

El estudio de fatiga permitió establecer que al igual que para el caso de las tensiones, el comportamiento del clavo Gamma es mejor que el de la placa DHS, ya que con dicho clavo se obtuvo mayor vida en ciclos.

De acuerdo con los estudios realizados, con ambas osteosíntesis se obtuvieron resultados aceptables, donde el clavo Gamma fue el de mejores resultados. No obstante, los estudios de las tensiones y de fatiga no deben ser la única consideración a la hora de escoger un fijador para su utilización en un paciente.

\section{RefERENCIAS}

Bayoglu R. y Okyar A.F. Implementation of boundary conditions in modeling the femur is critical for the evaluation of distal intramedullary nailing. Medical Engineering \& Physics, volumen 37 (número 11), 2015: 1053-1060.

Behrens B.A., Nolte I., Wefstaedt P., Stukenborg-Colsman C., Bouguecha A. Numerical investigations on the strain-adaptive bone remodelling in the periprosthetic femur: Influence of the boundary conditions. BioMedical Engineering OnLine, volumen 8 (número 1), 2009: 7.

Bergmann G. et al. Hip contact forces and gait patterns from routine activities. Journal of Biomechanics, volumen 34 (número 7) 22 de febrero, 2001: 859-871.

Buroni F., Commisso P., Cisilino A., Sammartino M. Determinación de las constantes elásticas anisótropas del tejido óseo utilizando tomografías computadas. Aplicación a la construcción de modelos de elementos finitos. Mecánica Computacional, volumen XXIII (número 28), Noviembre de 2004: 3009-3032.

Chang C.W., Chen Y.N., Li C.T., Peng Y.T., Chang C.H. Role of the compression screw in the dynamic hip-screw system: A finite-element study. Medical Engineering \& Physics, volumen 37 (número 12), 2015: 1174-1179.

Chen N.Z., Wang G., Guedes-Soares C. Palmgren-Miner's rule and fracture mechanics-based inspection planning. Engineering Fracture Mechanics, volumen 78 (número 18), 2011: 31663182.

Chen S.H., Chiang M.C., Hung C.H., Lin S.C., Chang H.W. Finite element comparison of retrograde intramedullary nailing and locking plate fixation with/without an intramedullary allograft for distal femur fracture following total knee arthroplasty. The Knee, volumen 21 (número 1), 2014: 224-231.

Estrada R., Ramos Y., Bosch J. Estudio biomecánico hueso-implante DHS y fijador externo en la consolidación de la fractura de cadera. Revista Cubana de Ortopedia y Traumatología, volumen 28 (número 1), enero-junio de 2014: 14-25.

Geraldes D.M. y Phillips A.T.M. A comparative study of orthotropic and isotropic bone adaptation in the femur. International Journal for Numerical Methods in Biomedical Engineering, volumen 30 (número 9), abril, 2014: 873-889.

Heller M.O. et al. Musculo-skeletal loading conditions at the hipduring walking and stair climbing. Journal of Biomechanics, volumen 34 (número 7), febrero de 2001: 883-893.

Kayabasi O. y Ekici B. Probabilistic design of a newly designed cemented hip prosthesis using finite element method. Materials \& Design, volumen 29 (número 5), 2008: 963-971.

Matityahu A., Schmidt A.H., Grantz A., Clawson B., Marmor M., McClellan R.T. The variable angle hip fracture nail relative to the Gamma 3: A finite element analysis illustrating the same stiffness and fatigue characteristics. Advances in Orthopedics, 20131-11, 2013.

Mohammad K., Zainudin E., Salit M., Zahari N., Ali A. Experimental determination of the fatigue behavior of austenitic 316L stainless steel under fatigue and creep-fatigue tests at high temperature. International Journal of Metal and Steel Research Technology, volumen 1 (número 1), 2013: 01- 11.

Müller C.M. y Cerrolaza M. Un nuevo método para la simulación de la estructura ósea mediante la versión $p$ de elementos finitos. Boletín Técnico, volumen 39 (número 3), 2001: 1-32.

Netterl F.H. Atlas de anatomía humana, Barcelona (España), 5a ed., Elsevier Health Sciences Spain, 2011, pp. 478-486.

Ojeda C.J. Estudio de la influencia de estabilidad primaria en el diseño de vástagos de prótesis femorales personalizadas aplicación a paciente específico, (tesis doctoral), Madrid, España, Universidad Politécnica de Madrid, Departamento de Ingeniería Mecánica y Fabricación, 2009, 342 p.

Orozco R. El ocaso de las placas. ¿Por qué se rompen los implantes? Revista de Ortopedia y Traumatología, volumen 45 (número 3), 2001: 177-182.

Pise U., Bhatt A., Srivastava, R. Influence of isotropic and orthotropic relationships on the accuracy of B-spline based heterogeneous graded finite element modeling of proximal femur. Computer - Aided Design \& Applications, volumen 9 (número 4), 2012: 549-569.

Ramos A. y Simões J.A. Tetrahedral versus hexahedral finite elements in numerical modelling of the proximal femur. Medical Engineering \& Physics, volumen 28 (número 9), 2006: 916-924.

Ramos Y., Estrada R., Bosch J. Empleo de elementos finitos en un estudio comparativo de dos sistemas de fijación interna para la fractura de cadera. Revista Cubana de Ortopedia y Traumatología, volumen 27 (número 2), 2013: 186-198. 
Rellán J. et al. Simulación del comportamiento mecánico de la fijación del fascículo posterolateral con tornillo biocompatible en reparaciones de ligamento cruzado anterior. Trauma, volumen 23 (número 2), 2012: 77-83.

Samiezadeh S., Tavakkoli-Avval P., Fawaz Z., Bougherara H. Biomechanical assessment of composite versus metallic intramedullary nailing system in femoral shaft fractures: A finite element study. Clinical Biomechanics, volumen 29 (número 7), 2014: 803-810.

Seral B., García M., Cegoñino J., Doblaré M., Seral F. Estudio biomecánico de la osteosíntesis intramedular en el tratamiento de las fracturas trocantéreas de cadera: Gamma y PFN. Revista Española de Cirugía Ortopédica y Traumatología, volumen 45 (número 05), 2001: 423-429.

Smith W.F. y Hashemi J. Fundamentos de la ciencia e ingeniería de materiales, México, 4a ed., McGraw-Hill, 2006, pp. 525-534.

Swanson S.A.V., Freeman M.A., Day W.H. The fatigue properties of human cortical bone. Medical and Biological Engineering, volumen 9 (número 1), 1971: 23-32.

Tavakkoli-Avval, P., Samiezadeh S., Klika V., Bougherara H. Investigating stress shielding spanned by biomimetic polymercomposite vs. metallic hip stem: A computational study using mechano-biochemical model. Journal of the Mechanical Behavior of Biomedical Materials, volumen 4156, (número 67), 2015.

Taylor D. Fatigue of bone and bones: an analysis based on stressed volume. Journal of Orthopaedrc Research, volumen 16 (número 2), 1998: 163-169.

Trabelsi N. y Yosibash Z. Patient-specific finite-element analyses of the proximal femur with orthotropic material properties validated by experiments. Journal of Biomechanical Engineering, volumen 133 (número 6), 2011: 1-12.

Trębacz H., Zdunek A., Cybulska J., Pieczywek P. Effects of fatigue on microstructure and mechanical properties of bone organic matrix under compression. Australas Phys Eng Sci Med, volumen 36 (número 1), 2013: 43-54.

Viceconti M., Bellingeri L., Cristofolini L., Toni A. A comparative study on different methods of automatic mesh generation of human femur., Medical Engineering \& Physics, volumen 20 (número 1), 1998: 1-10.

Vigil F., Guerrero M., Jorge J., Rivera S., Iglesias A. Diseño y validación a fatiga de un vástago de revisión de prótesis de cadera empleando el método de los elementos finitos. Anales de Mecánica de la Fractura, volumen 28 (número 2), 2011: 817822. 


\section{Citación sugerida:}

\section{Citación estilo Chicago}

Ramos-Botello, Yoan Manuel, René Misael Arias-Hidalgo, Jorge Alexander Bosch-Cabrera, Roberto Andrés Estrada-Cingualbres. Análisis numérico comparativo del comportamiento a fatiga de dos osteosíntesis utilizadas en el tratamiento de la fractura de cadera 31 A1 1. Ingeniería Investigación y Tecnología, XVIII, 04 (2017): 445-455.

\section{Citación estilo ISO 690}

Ramos-Botello Y.M, Arias-Hidalgo R.M, Bosch-Cabrer J.A, EstradaCingualbres R.A. Análisis numérico comparativo del comportamiento a fatiga de dos osteosíntesis utilizadas en el tratamiento de la fractura de cadera 31 A1 .1. Ingeniería Investigación y Tecnología, volumen XVIII (número 4), octubre-diciembre 2017: 445-455.

\section{Semblanzas de loS autores}

Yoan Manuel Ramos-Botello. Ingeniero mecánico por la Universidad de Holguín en 2008. Obtuvo su título de master en ciencias en el 2011 por la misma institución. Actualmente se desempeña como profesor de elementos finitos en la carrera de ingeniería mecánica de la Universidad de Granma. Sus trabajos de investigación se centran en el área de la biomecánica computacional y el CAD/CAE.

René Misael Arias-Hidalgo. Se graduó con título de oro como ingeniero mecánico en el 2016 por la Universidad de Granma. Actualmente se prepara para impartir clases de ingeniería mecánica en dicha universidad.

Jorge Alexander Bosch-Cabrera. Ingeniero mecánico desde el 2007 por la Universidad de Holguín. Obtuvo el título de master en ciencias en la misma Universidad (2011). Actualmente se desempeña como profesor de elementos de máquinas en la carrera de ingeniería mecánica de la Universidad de Granma. Sus trabajos de investigación se centran en el área de la biomecánica computacional y el CAD/CAE.

Roberto Andrés Estrada-Cingualbres. Graduado como ingeniero mecánico en Rusia. Obtuvo su título de PhD en 1999 en la Universidad de Holguín, donde se desempeña actualmente como profesor titular en la carrera de ingeniería mecánica y vicedecano de investigaciones y posgrado. Ee especialista en el área de elementos finitos, ha desempeñado importantes investigaciones en el área del análisis estructural y la biomecánica computacional. 
DOI: http://dx.doi.org/10.22201/fi.25940732e.2017.18n4.039 